\title{
IMPROVING THE SENSITIVITY OF THE OKP VISUAL FIELD SCREENING TEST WITH A BLUE STIMULUS ON A DARK BACKGROUND
}

\author{
IZAK F. WESSELS ${ }^{1}$ AND RUVDEEP S. RANDHAWA ${ }^{2}$ \\ Loma Linda, California; and St Louis, Missouri
}

\begin{abstract}
SUMMARY
The standard oculokinetic perimetry test (OKP) was modified to present a light blue stimulus on a dark background (MOKP) to determine whether the sensitivity and specificity for detecting glaucomatous visual field loss could be improved. Thirty-five adult glaucoma patients (70 eyes) self-administered both tests and the results were correlated with the loss of retinal sensitivity on the Octopus IV program 38. The MOKP detected $18 \%$ more true scotomatous loci (more than $15 \mathrm{~dB}$ loss of attenuation) than the standard OKP $(p<0.0001)$. The gain was due to $37 \%$ fewer false negatives $(p<0.0001)$, but with a doubling of the false positives rate $(p<0.049)$. With a disease prevalence of approximately $2 \%$, the MOKP and OKP would respectively miss $15 \%$ or $30 \%$, and include 14 or 9 normals for each diseased individual. This relatively simple modification may further improve the OKP for detecting glaucoma.
\end{abstract}

Glaucoma is a major public health problem: after cataract and trachoma it is the third most common cause of blindness worldwide and the leading cause of blindness in the United States. ${ }^{1.2}$ Primary open angle glaucoma occurs in about $2 \%$ of whites, but is allegedly 4-5 times more frequent in blacks. ${ }^{3}$ Early detection is critical because despite effective treatment being available, symptoms are subtle and optic nerve damage results in permanent visual field loss. ${ }^{1}$ Although detecting defects in the visual field is the most reliable method for identifying glaucoma, the need for expensive equipment, skilled technicians, and length of time taken makes it unsuitable for general screening. ${ }^{4.5}$

From: ${ }^{1}$ Department of Ophthalmology, Loma Linda University, California; and ${ }^{2}$ Washington University, St Louis, Missouri, USA.

Correspondence to: Izak F. Wessels, Omni Eye Services, 1949 Gunbarrel Road, Suite 280, Chattanooga, TN 37421, USA. Telephone: +1 (423) 885 1666. Fax: +1 (423) 8920787.
The Oculokinetic Perimeter Test (OKP) has been developed to make visual field screening possible: a simple, inexpensive, and self-administered (in trustworthy witnesses) visual field screening device that relies on eye movements to project a stimulus onto different areas of the visual field. ${ }^{6-9}$ It consists of a piece of firm white card with a central $1.5 \mathrm{~mm}$ black target surrounded by 26 numbers in a spiral pattern that subtend the central $30^{\circ}$ visual field. Directions on the card instruct the subject to cover one eye and then to look directly at each of the numbers. If the central black stimulus disappears, a defect has been detected. The examiner is encouraged to cover and uncover the stimulus to exclude inconsistent results.

A dark stimulus is an unconventional method of performing perimetry. ${ }^{8}$ Although promoted for large-scale population screening, its lack of sensitivity (detecting defects of more than $16-20 \mathrm{~dB}$ as compared with age-normal subjects) may make it less useful for early detection of disease. ${ }^{9-12}$

A blue stimulus may detect glaucomatous field loss and nerve fibre layer damage earliest, since the large ganglion cells with a preferential sensitivity to blue are damaged first. ${ }^{13}$ Detecting a defect to blue may therefore be more sensitive and specific than a loss to a white or, in the case of the OKP test, a black stimulus. We modified the OKP to present a light blue stimulus on a dark background instead of a black stimulus on a white background.

\section{MATERIALS AND METHODS}

The modified OKP test (MOKP) is identical in size to the OKP test and is a photographically reproduced blue 'negative', with a dark background and light blue central stimulus (Fig. 1). The modified chart was attached with hinges to one side of the OKP chart to permit superimposition of the MOKP while using the same occluder (Fig. 2). The central light blue stimulus best matched with $2995 \mathrm{C}$ and the background with $282 \mathrm{C}$ of the Pantone Color Selector

Eye (1996) 10, 620-625 C 1996 Royal College of Ophthalmologists 


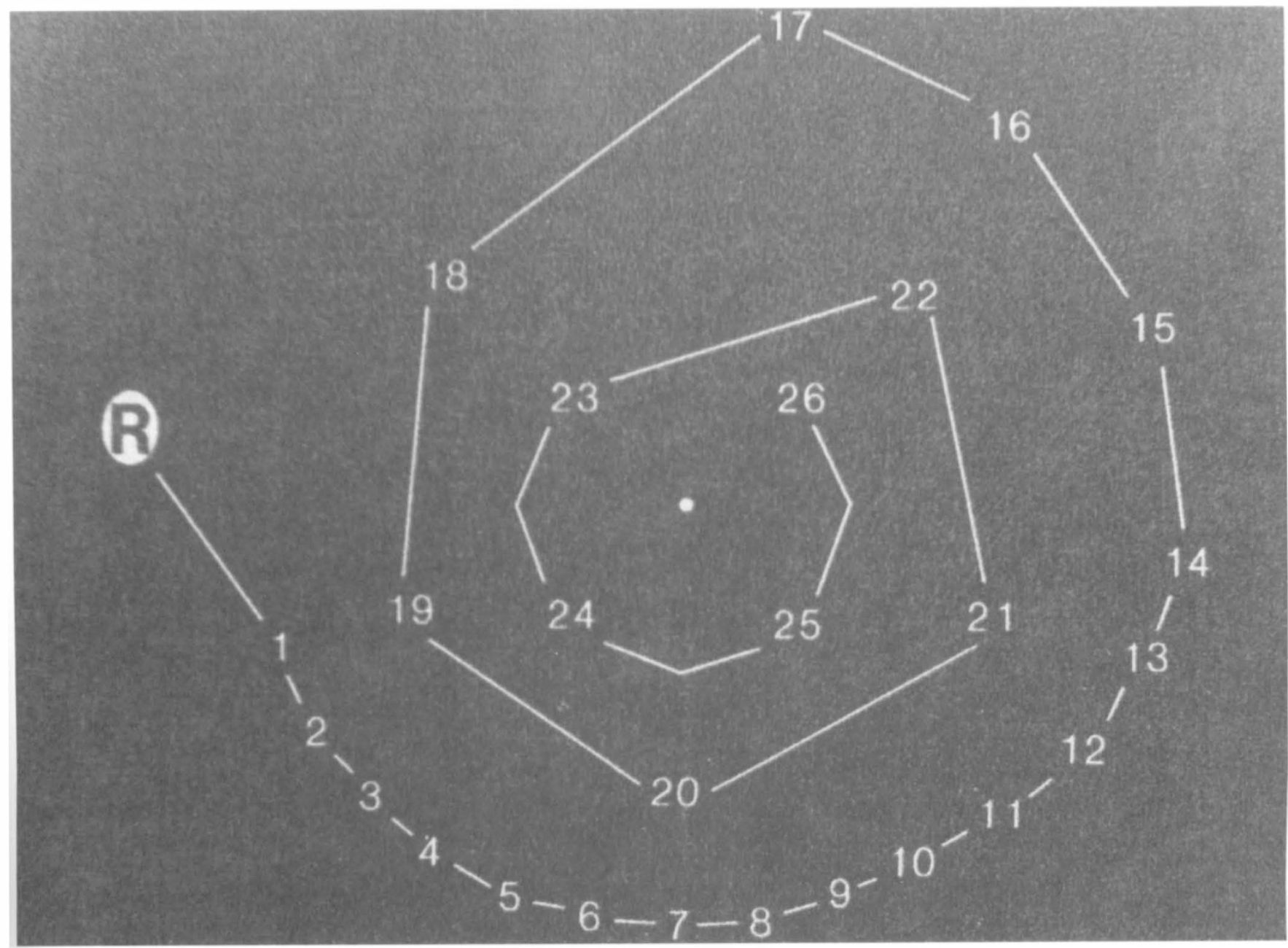

Fig. 1. The pattern of a concentric spiral of numbers around a central stimulus is the significant element of the OKP. If the central test dot disappears while the subject is looking at a specific number, a scotoma has been detected. The modified OKP consist of a light (blue) stimulus on a dark background to increase the sensitivity.

1000 (Pantone, Carlstadt, NJ). Measuring the reflectance of the stimulus and background yielded respectively 0.78 and 1.56 optical units with a model TR 924 Macbeth Densitometer (Macbeth, NY).

The Institutional Review Board for Loma Linda University approved the protocol for this project and its requirements were strictly adhered to. All subjects were recruited from a consecutive cohort of patients attending the glaucoma clinic at Loma Linda University Eye Medical Group. Exclusion criteria included: unwillingness to participate after receiving the informed consent form; corrected visual acuity less than 20/40; excessive frailty; or unreliable Octopus test results. All subjects underwent conventional computerised perimetry with the Octopus 500EZ, using program 38, just before or just after taking the MOKP and OKP tests. If mydriatic drops had been instilled, the Octopus and both OKP tests were performed under the same pupillary condition. The ambient illumination from ceiling-mounted diffused fluorescent lights was 880 foot-candles and the light reflected off the OKP chart measured 13 lux. All subjects wore their spectacles (with bifocals) and chose which eye was tested first. After the procedure had been explained, the patients selfadministered both tests with the examiner present, but without interruption. The first test presented (MOKP or OKP) was randomised for the first as well as for the second eye. As the test protocol suggests, when a defect was detected at a given locus, the same locus was re-tested to confirm reproducibility. ${ }^{6-9}$ The results were scored on the sheets supplied.

From the Octopus printout grey scale with steps of $5 \mathrm{~dB}$ attenuation, the least decibel attenuation determined the retinal sensitivity corresponding to each OKP test locus. Loci with $15 \mathrm{~dB}$ or less attenuation were arbitrarily defined as 'scotomatous', with glaucoma damage. The OKP ought to detect a defect, i.e. yield a positive result. Likewise, loci with more than $15 \mathrm{~dB}$ of attenuation were defined as 'nonscotomatous', or normal. At these loci the OKP ought not to detect a defect, i.e. yield a negative result. The reliability of the tests were judged by 


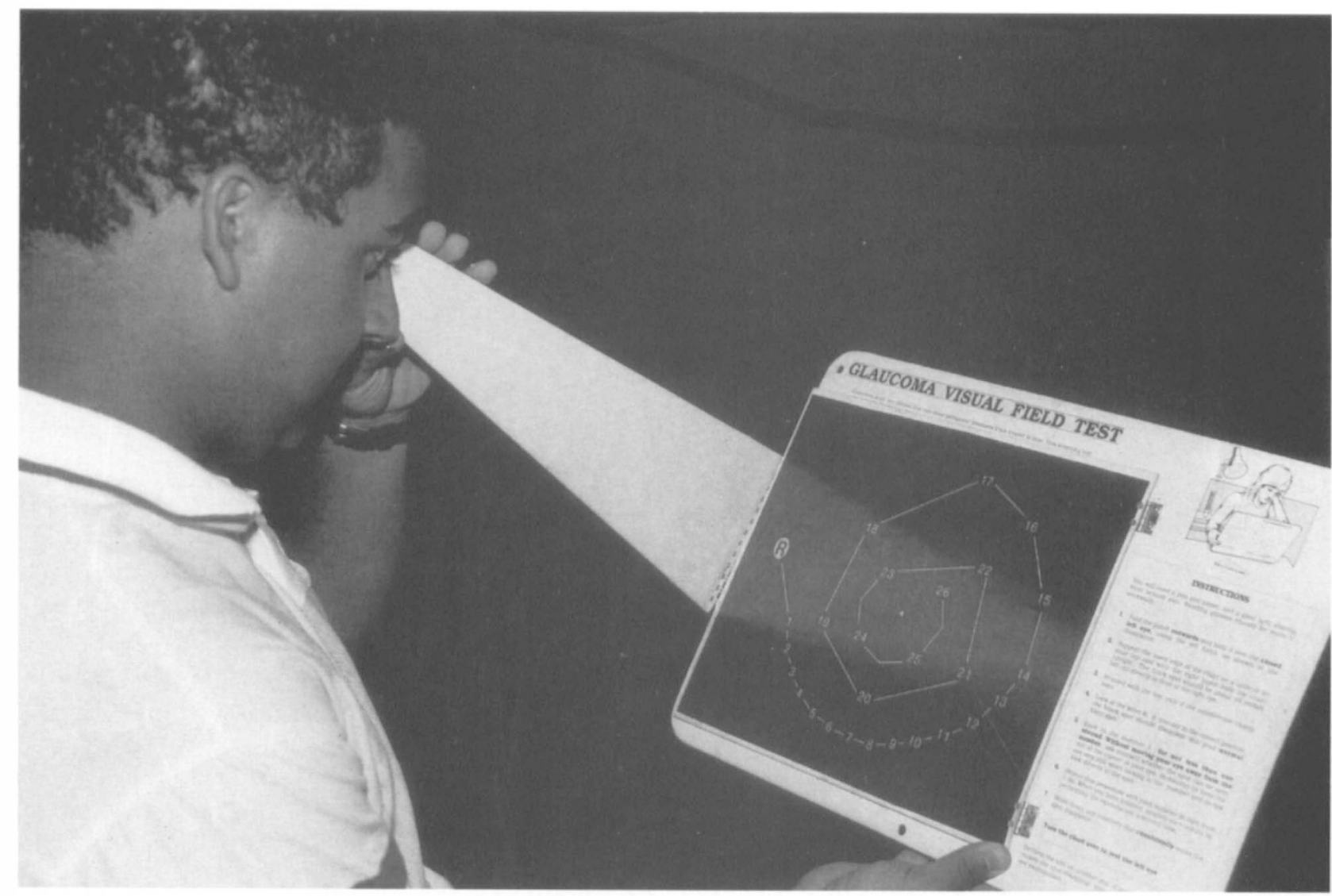

Fig. 2. The occluder attached to the OKP test target serves to control the angle the chart subtends with respect to the eye. By fixating on each number, the central stimulus successively tests different loci within the central $30^{\circ}$ visual field. In this photograph, the subject is using the modified test chart.

determining whether a positive or negative response at each locus was true or false: a true positive response detected a defect in a 'scotomatous' Octopus locus; a false positive response detected a defect in a 'normal' Octopus locus; a false negative did not detect a defect where the Octopus showed a scotoma; and a true negative detected no defect where the Octopus found a normal retinal sensitivity. All calculations were performed on a PC spreadsheet program (Microsoft Excel) and the statistical analysis was performed with WinStar, using the paired $t$-test.

\section{RESULTS}

Thirty-five patients met the criteria for inclusion and 70 eyes were tested. There were 27 whites, 2 blacks and 6 hispanics. Seventeen were female and 18 male, with a mean age of 56 years (range 19-82 years). The results are presented in Table I, and graphically in Fig. 3. When the results were separated into left and right eyes, the same trend was apparent as in the pooled results, with the sample size influencing the magnitude of statistical significance.

True positives. The SOKP test detected a mean of $5.94(\mathrm{SD}=5.96$, range $0-21)$ defects and the modified test 7.00 ( $\mathrm{SD}=6.43$, range $0-18)$, i.e. there was a mean gain of 1.06 true positive results
(18\%) with the MOKP. This improvement was statistically highly significant $(p<0.0001)$ using the paired $t$-test. When the results are separated for right and left eyes, the mean gain (additional defects detected) is respectively $21 \%$ and $17 \%$ with the modified test.

False positives. The SOKP test yielded a mean of 0.11 (SD $=0.36$, range $0-1$ ) and the modified test 0.24 (SD 0.43, range 0-2) false positive results. This deterioration was statistically significant $(p \leqslant 0.049)$ with the paired $t$-test.

False negatives. The standard test produced a mean of 2.93 (SD $=2.77$, range $0-7)$ and the modified test $1.84(\mathrm{SD}=1.95$, range $0-9)$ false negative results. The difference of 1.09 was statistically highly significant $(p<0.0001)$ with the paired $t$ test. The pooled results showed a gain of $37 \%$ fewer false negatives; and respectively $42 \%$ and $35 \%$ if the right and left eyes are separated.

Mean attenuation for positive stimuli. The mean attenuation for all defects detected was $14.45 \mathrm{~dB}$ $(\mathrm{SD}=8.23)$ for the modified test and $15.21 \mathrm{~dB}$ $(\mathrm{SD}=8.28)$ with the standard test; the difference was not statistically significant with the $t$-test $(t=0.545)$.

Mean minimum attenuation for negative stimuli. 
Table I. The mean number of defects detected with OKP and MOKP

\begin{tabular}{|c|c|c|c|c|}
\hline & OKP & MOKP & $\mathrm{MOI}$ & \\
\hline & Mean (SD; range) & Mean (SD; range) & Difference (\% change) & Significance \\
\hline True positi & (mean defects dete & more than $15 \mathrm{~dB}$ att & & \\
\hline Right eyes & $6.63(6.18 ; 0-21)$ & $8.00(6.62 ; 0-18)$ & $1.37(+21 \%)$ & $(p=0.0006)$ \\
\hline Left eyes & $5.26(5.75 ; 0-21)$ & $6.00(6.17 ; 0-18)$ & $0.74(+17 \%)$ & $(p=0.0005)$ \\
\hline Both eyes & $5.94(5.96 ; 0-21)$ & $7.00(6.43 ; 0-18)$ & $1.06(+18 \%)$ & $(p<0.0001)$ \\
\hline False negat & $s$ (mean defects det & oite $15 \mathrm{~dB}$ or less of & tion) & \\
\hline Right eyes & $2.91(2.85 ; 0-6)$ & $1.69(1.68 ; 0-9)$ & $-1.22(-42 \%)$ & $(p=0.0009)$ \\
\hline Left eyes & $2.94(2.73 ; 0-7)$ & $2.00(2.21 ; 0-9)$ & $-0.94(-35 \%)$ & $(p=0.0004)$ \\
\hline Both eyes & $2.93(2.77 ; 0-7)$ & $1.84(1.95 ; 0-9)$ & $-1.09(-37 \%)$ & $(p<0.0001)$ \\
\hline False positi & $s$ (mean defects dete & ite more than $15 \mathrm{~dB}$ & ation) & \\
\hline Right eyes & $0.14(0.36 ; 0-1)$ & $0.23(0.43 ; 0-1)$ & $0.09(+64 \%)$ & $(p=0.3244)$ \\
\hline Left eyes & $0.11(0.37 ; 0-1)$ & $0.26(0.44 ; 0-2)$ & $0.15(+136 \%)$ & $(p=0.0831)$ \\
\hline Both eyes & $0.11(0.36 ; 0-1)$ & $0.24(0.43 ; 0-2)$ & $0.13(+118 \%)$ & $(p<0.0488)$ \\
\hline
\end{tabular}

True positives, false negatives or false positives are so defined according to whether the Octopus 500 EZ visual field showed an attenuation of $15 \mathrm{~dB}$ at that locus.

The minimum attenuation of defects not detected provides a measure of the threshold values for the stimulus. Comparing the negative stimuli revealed a mean minimum attenuation (i.e. most intense Octopus stimulus) of 19.23 ( $\mathrm{SD}=6.8$ ) for the MOKP test, and $16.06(\mathrm{SD}=8.57)$ for the OKP test, a difference which was statistically highly significant $(p=0.0078)$ on the $t$-test $(t=2.42)$.

\section{DISCUSSION}

Both tests showed excellent agreement with the Octopus findings, despite the potential errors common to all screening tests: lack of sensitivity yielding false negative results, or a lack of specificity yielding false positives. ${ }^{5}$ A low specificity may be preferable to a low sensitivity if the disease has a relatively high prevalence, as is the case with glaucoma. Visual field tests measure differential light sensitivities or the ability to detect a just noticeable difference between a stimulus and the background intensity. The relative stimulus intensity from a printed surface can be approximated by illuminance and surface densitometry. Densitometry units (as specified by ISO 5/3) are the logarithm of the inverse of the reflectance, with an absolutely black surface reflecting the least, and therefore the inverse yields the maximum value of

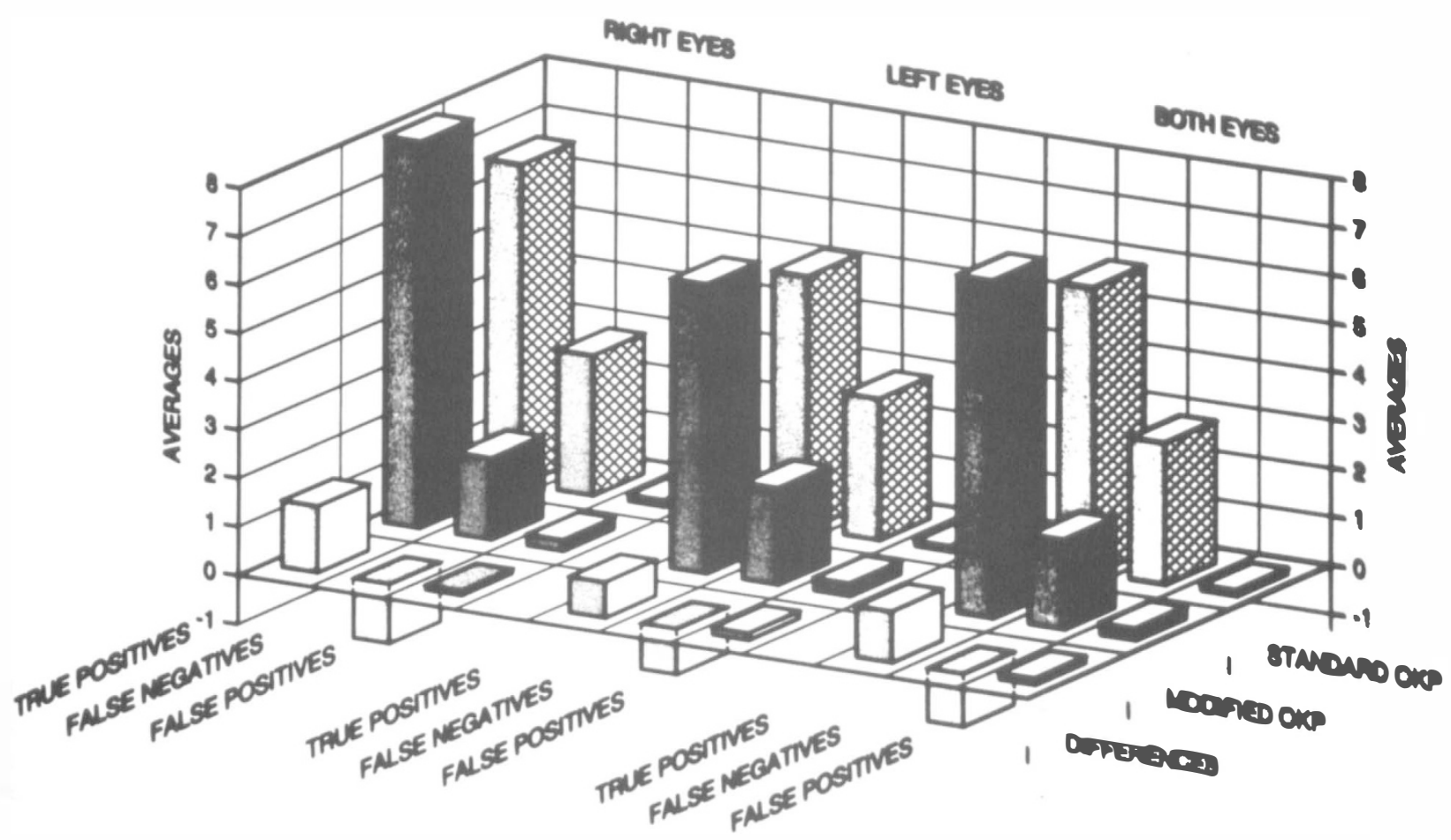

Fig. 3. This graph reproduces the numerical results of Table I, or the mean number of defects detected respectively for right, left and both eyes. The columns correspond to defects correctly detected (true positives); defects not detected (false negatives); and lastly defects detected in loci without a $15 \mathrm{~dB}$ loss of attenuation on the Octopus test (false positives). The front row (nearest) columns correspond to the differences between the middle dark columns (MOKP) and the rear columns (OKP). 
1.80, while a more reflecting surface yields a lesser inverse of reflectance. The MOKP dark background measured 1.56 and the lighter blue central stimulus 0.78 reflectance units, i.e. reflected light is attenuated by 10 to the power 1.56 or 0.78 . The difference between these two values is a measure of the differential contrast: 0.78 or $78 \mathrm{~dB}$. With the OKP the white background measured 0.10 and the dark target 1.75 reflectance units, yielding a relative difference of 1.65 or $165 \mathrm{~dB}$. This will markedly influence the luminance (Weber) contrast that probably was the mechanism for the modified test's sensitivity. Scotomata on the Octopus perimeter are quantitated by less attenuated (i.e. brighter) stimuli required to elicit a response. There was no difference in the mean attenuation of all defects detected between the two tests, as may be expected; averaging a wide range of responses obscures minor differences. When the least attenuations at loci not detecting a defect were averaged, the MOKP shows $19.23 \mathrm{~dB}$ and the OKP $16.06 \mathrm{~dB}(p<0.01)$, demonstrating that the MOKP presents a less supramaximal stimulus. Amsler grids using black backgrounds have been recommended to enhance retinal sensitivity. ${ }^{14}$

The standard OKP test presents a dark stimulus on a light background - an unconventional testing methodology when compared with other visual field tests. ${ }^{8}$ However, a dark stimulus may be less visible (i.e. be less of a supramaximal stimulus) and less dependent on ambient lighting while preferentially stimulating the off-centre ganglion cells. ${ }^{15}$ These cells comprise a relatively small percentage $(25-40 \%)$ of the cells in the central visual field and are therefore more likely to show defects after glaucoma injury. Since only 26 stimuli are presented, each additional defect detected is significant, the more so in early cases with the visual field still relatively intact. Our subjects with known glaucomatous damage showed a mean of six true positive defects with the OKP; and with the MOKP they gained one more. This increase in sensitivity of approximately $20 \%$ was due to having on average one fewer false negative. However, there was an increase in the mean false positive rate: with the MOKP $0.24(\mathrm{SD}=0.43$, range $0-2)$ as compared with $0.11(\mathrm{SD}=0.36$, range $0-1)$ with the OKP. This translates into one defect inappropriately detected every four or nine patients, respectively. This doubled false positive rate is of concern, since in every 1000 screened individuals, respectively 250 and 110 would be selected. With a disease prevalence of $2 \%$, the remaining 750 and 890 would respectively include 15 and 17 individuals with glaucoma. Considering the test sensitivities (the MOKP missed 2 of 9 true scotomata and the OKP 3 ) respectively 12 of 15 and 11 of 17 would be identified. If those individuals with glaucoma in the group of false positives (respectively 5 and 2) are included, the final ratio becomes 1 glaucoma patient identified respectively every 14 and 9 false positives.

The MOKP would miss 3 and the OKP 6 of the original $20(2 \%$ of 1000$)$ with a reliability of $85 \%$ and $70 \%$ respectively. Basing the calculation on the test findings yields very similar results: of 9 loci deemed scotomatous, the MOKP missed 2 and the OKP 3, a false negative ratio of $22 \%$ ( 2 of 9 ) and $33 \%$ ( 3 of 9 ) or a reliability of $88 \%$ and $68 \%$. All screening tests for diseases with low prevalence will identify more false positives than truly diseased individuals (specificity) and also miss some diseased individuals (sensitivity). ${ }^{5}$ Those making policy decisions in health care may increasingly consider the cost-tobenefit ratio of all procedures, as well as the cost of not detecting a disease which will require similar simplified ratios for other screening tests.

Whether one missed stimulus is sufficient to refer a subject for further evaluation is not clear: on average our patients had 9 of the 26 loci scotomatous by the $15 \mathrm{~dB}$ definition. There were too few false positive loci in our sample to detect differences in retinal sensitivity between the two tests. Future cvaluations in a larger clinical trial may help determine the ageand population-appropriate stimulus intensity which is most likely to distinguish between a false or a true response. $^{16}$

Other suggested improvements to the OKP include replacing some test loci near the macula with more in the nasal step area, as well as using a neutral density filter $0.3 \log$ units less than that which extinguishes a peripheral stimulus. ${ }^{17,18}$ Our highest positive yield was from loci 1 to $17 ; 18$ to 26 contributed only one-seventh of all defects with the OKP and one-third with the MOKP. This corroborates previous reports that suggest modifying the stimulus pattern. ${ }^{17}$ The original OKP is indeed no longer available, but has been replaced by a unit which allows the rotation of various stimuli of different size and contrast density into the central test area (Clement Clark, personal communication 1994).

We believe that as simple a change as colour and relative intensity of the stimulus and background improves the OKP sensitivity, with a gain in reliability but at the expense of an increased false positive rate. Our subjects spontaneously affirmed that although both tests were very easy to selfadminister, the light blue stimulus was more obviously 'missing' against a dark background than the black stimulus on the white background. These modifications require further evaluation as a part of a larger glaucoma screening programme before being generally implemented.

This work was supported by The California Museum of Science and Industry, Los Angeles, and The Inland Eye Institute, Loma Linda University, Loma Linda, California. 
We are indebted to Dr Zimmerman of the Loma Linda School of Health for statistical assistance.

Key words: Glaucoma, Perimetry, Reliability, Screening, Sensitivity, Specificity.

\section{REFERENCES}

1. Hart WM Jr. The epidemiology of primary open-angle glaucoma and ocular hypertension. In: Ritch R, Shields MB, Krupin T, editors. The glaucomas. St Louis: CV Mosby, 1989:789-95.

2. Vision problems in the US: facts and figures. New York: National Society to Prevent Blindness, 1981:1.

3. Tielsch JM, Sommer A, Katz MS, Royall AM, Quigley HA, Javitt J. Racial variation in the prevalence of primary open-angle glaucoma. JAMA 1991;3:369-74.

4. Keltner JL, Johnson CA. Current status of automated perimetry. Is the ideal automated perimetry available? Arch Ophthalmol 1983;28:206-26.

5. Gottleibb LK, Schwartz B, Panker SG. Glaucoma screening: a cost-effectiveness analysis. Surv Ophthalmol 1983;28:206-26.

6. Alvarez E, Damato BE, Jay JL, McClure E. Comparative evaluation of oculokinetic perimetry in glaucoma. Br J Ophthalmol 1988;72:258-62.

7. Damato BE, Ahmed J, Alvarez E, Allen D, McClure E, Jay JL. Oculokinetic perimetry. In: Mills KB, editor. Glaucoma. New York: Pergamon Press, 1988:84-91.

8. Mutlukan E, Damato BE. The dark perimetric stimulus. Br J Ophthalmol 1992;76:264-7.

9. Damato BE, Chyla J, McClure E, Jay JL. A hand-held
OKP chart for the screening of glaucoma: preliminary evaluation. Eye 1990;4:632-7.

10. Vernon SA, Quigley HA. A comparison of the OKP visual field screening test with the Humphrey field analyser. Eye 1992;6:521-4.

11. Wishart PK. Oculokinetic perimetry compared with Humphrey visual field analysis in the detection of glaucomatous visual field loss. Eye 1993;7:113-21.

12. Henson DB, Dix SM. Evaluation of the Freedman Visual Field Analyser Mark II. II. Results from a population with induced visual field defects. $\mathrm{Br} \mathrm{J}$ Ophthalmol 1984;68:463-7.

13. Hugulstone CE, Vernon SA. Use of a blue filter in visual field analysis. Br J Ophthalmol 1991;75:155-7.

14. Wolfe KA, Sadun AA. Threshold Amsler grid testing in diabetic retinopathy. Graefes Arch Clin Exp Ophthalmol 1991;229:219-23.

15. Saito T. Physiological and morphological differences between on- and off-centre bipolar cells in the vertebrate retina. Vision Res 1987;27:135-42.

16. Mutlukan E, Damato BE, Jay JL. Clinical evaluation of a multi-fixation campimeter for the detection of glaucomatous visual field loss. $\mathrm{Br} \mathrm{J}$ Ophthalmol 1993;77:332-8.

17. Stirling RJ, MacLeod JDA, Vernon SA. A new chart to improve the efficiency of glaucoma detection by oculokinetic perimetry. Eye 1994;8:121-4.

18. Vernon SA, Quigley HA. Improving the sensitivity of the OKP visual field screening test with the use of neutral density filters. Eye 1994;8:406-9. 